\title{
Research on LTE/Wi-Fi Coexistence in Unlicensed Bands
}

\author{
Peng Li, Shou-liang Yang**, Hong-ping Gan, \\ Bao-liang Yang, Ling-gang Zeng, Qin-guo Hu \\ School of Electronic and Electrical Engineering \\ Chongqing University of Arts and Sciences \\ Chongqing, China \\ lipeng663073@cqu.edu.cn
}

\begin{abstract}
Spectrum sharing is a highly-efficient method to solve the problem of the exponential capacity demand increase in wireless communication. Long Term Evolution(LTE) and Wi-Fi are two of the most remarkable wireless technologies nowadays. And it is of great importance to research on the coexistence of the two technologies. When the interference level goes below a certain threshold, the channel is accessible to communication nodes while operating Wi-Fi network. As a result, it exerts significant influence on Wi-Fi operation with LTE coexisting. The work introduces some schemes in order to realize LTE/Wi-Fi coexistence. As demonstrated in the test results, the spectral efficiency of LTE is higher than that of Wi-Fi in unlicensed band. Therefore, it is reasonable for LTE to compete for using unlicensed band. Simulation results show that there is a significant increase in the cell throughput and the communication rate of users by aggregating licensed band and unlicensed band in LTE network. Meanwhile, it turns out to be a slight lower performance of Wi-Fi. The range of the performance degradation of Wi-Fi is narrower than that of the performance promotion of LTE, thus achieving the performance promotion of overall system.
\end{abstract}

Keywords-LTE/Wi-Fi coexistence; unlicensed bands; channel selection; supplement down link

\section{INTRODUCTION}

With the rapid growth of mobile communication terminals, mobile data traffic also shows a steeply increasing trend. Cisco forecast, up to 2018, the number of global mobile terminals will be 1.4 times of the world's population; Global mobile data traffic will be 11 times in 2013, and the compound annual growth rate is $61 \%$ [1]. In addition to the rapid growth of terminals and data traffic, there will be a dramatic expansion in the scenarios, application field, and business types of mobile communication. In the future, mobile communication will have characteristics like large capacity, high speed, density and mobility as well as low delay. To achieve the mobile communication with above characteristics, mobile communication technology should make further improvements basing on the fourth generation mobile communication (4G), so that it can meet the demand of the fifth generation mobile communication $(5 \mathrm{G})$.

Required by the fifth generation mobile communication, more spectrums should be provided. International Telecommunications Union (ITU) says that the use efficiency

This paper is funded by: Scientific and Technological Research Program of Chongqing Municipal Education Commission (KJ1501107, KJ1501105)

Corresponding author: Shou-liang Yang of wireless spectrum should be increased by 10 times to meet the growing demand of mobile broadband users [2]. Some important alternative technical specifications are the fourth Generation mobile communication technology specification Long Term Evolution (LTE) and LTE-advanced introduced by the 3 rd Generation Partnership Project (3 GPP) [3].

As shown in Fig. 1, 3GPP members, Qualcomm, Huawei, China Mobile Communications Corporation and other wellknown companies at home and abroad, set the aggregated spectrum of LTE and LTE-Advanced in unlicensed spectrum. Spectrum available for aggregation, however, is not assigned to the exclusive use of mobile communication. The fragmented and disperse spectrum have to be shared with other wireless communication technologies $[4,5]$, such as the Wi-Fi technology, the most representative one of Wireless Local Area Network (WLAN).

The paper is organized as follows: Section II presents LTE/Wi-Fi coexistence schemes. Simulation tool and deployment scenario are presented in Section III. Simulation results are given in Section IV, while conclusions are given in Section V.

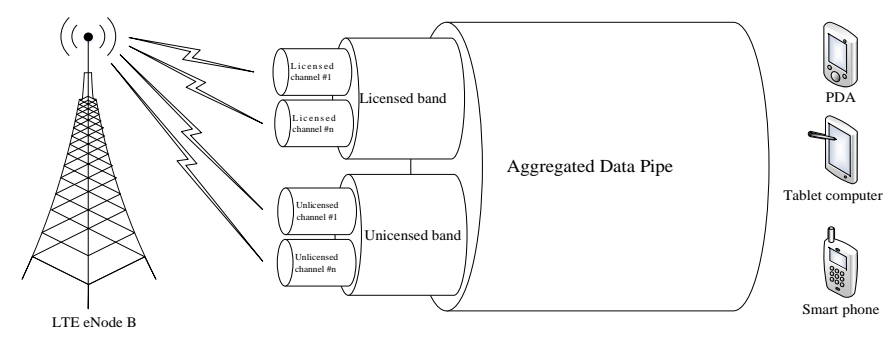

Fig. 1. The combination of unlicensed band and licensed band.

\section{LTE/WI-FI COEXISTENCE SCHEMES}

Research indicates that, in unlicensed spectrum, LTE and $\mathrm{Wi}-\mathrm{Fi}$ are the most competitive wireless communication technologies with good market prospects $[6,7]$. Therefore, it is necessary to solve the problem of the co-channel interference between the two technologies. As Wi-Fi is lack of the control mechanism of interference, researchers domestic and overseas mainly improve the LTE, so as to solve the problem of the coexistence of LTE and Wi-Fi in unlicensed spectrum [8]. To achieve the coexistence of LTE and $\mathrm{Wi}-\mathrm{Fi}$ in unlicensed 
spectrum, we can adopt the technical solutions as follows: Channel Selection, as shown in figure 2, LTE choose the Clean channel as the working channel [9]; LTE and Wi-Fi share a channel via time-division multiplexing $[10,11]$, as shown in figure 3 , Wi-Fi adopts the CSAT (carrier sensing the adaptive transmission) to monitor channels to be used, and Wi-Fi will use the channels if the channels are not occupied in a period of time. Wi-Fi monitors channels periodically. And if the channel is used, the Wi-Fi back off after a while, then access the channel again. When the network load is heavy, LTE adopt the SDL (Supplement the Down Link) technology and use the unlicensed band as a supplement to provide more bandwidth for downlink transmission [12].

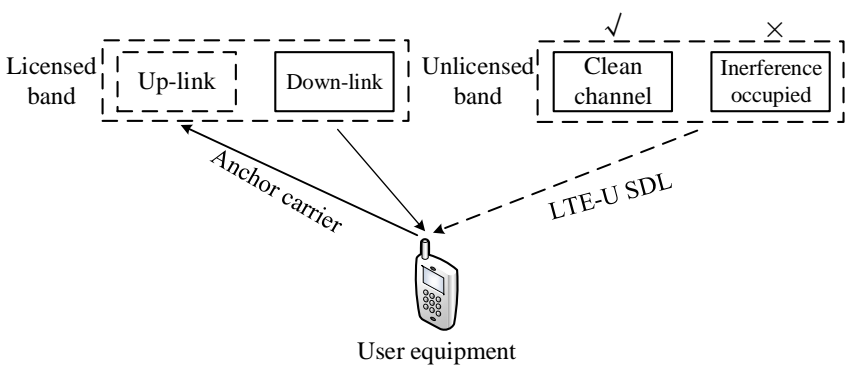

Fig. 2. Channel selection for LTE-Unlicensed band.

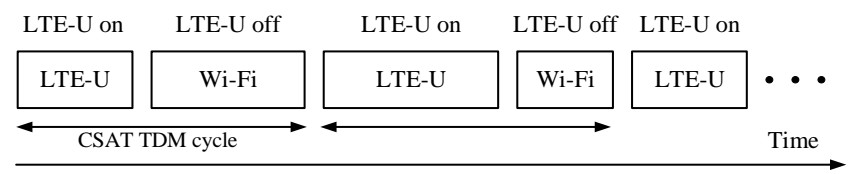

Fig. 3. LTE-Unlicensed band and Wi-Fi to share the same channel.

\section{SIMULATION TOOL AND DEPLOYMENT SCENARIO}

Simulation uses Quadrature Phase Shift Keying (QPSK) modulation. After synchronization and Fast Fourier Transformation (FFT), the receiver obtains a frame data. The positioning error of the FFT window leads to the rotation of constellation point. While the Inter-Carrier Interference (ICI) leads to cloud divergence of constellation. As a result, the band-shaped ring is formed. After the phase correction is adopted, constellation points of the received data are gathered again in positions of signal space.

We evaluate LTE/Wi-Fi coexistence in an indoor environment which consists of 20 single floor rooms. As shown in Fig. 4, we can see the relevant spatial information in the scenario. According to indoor propagation model [13], we model path loss and shadowing. And we use Rayleigh fading to represent multipath propagation effects which are exerted on information interfering and bearing signals. Table I summarizes the simulation parameters.

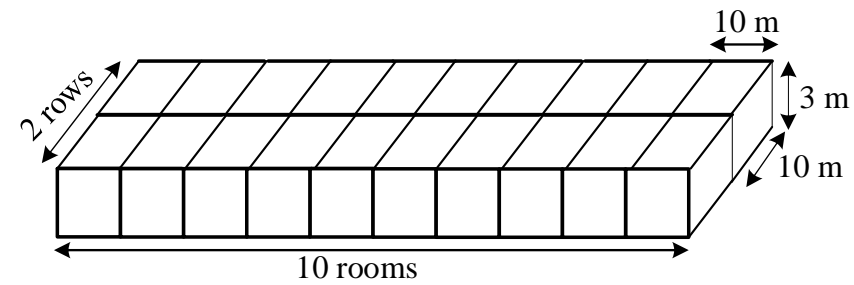

Fig. 4. Single floor office scenario: 10 rooms per row.
TABLE I D DEPLOYMENT SCENARIO AND SIMULATION PARAMETERS

\begin{tabular}{ll}
\hline \multicolumn{1}{c}{ Parameter } & \multicolumn{1}{c}{ Value } \\
\hline Scenario & Dual stripe single floor \\
System bandwidth & $20 \mathrm{MHz}$ \\
Center frequency & $5.8 \mathrm{GHz}$ \\
Maximum transmission power & $20 \mathrm{dBm}$ \\
LTE power control & Closed loop \\
eNode B height & $1.0 \mathrm{~m}$ \\
Access points height & $1.0 \mathrm{~m}$ \\
User equipment height & $1.5 \mathrm{~m}$ \\
Wi-Fi Stations height & $1.5 \mathrm{~m}$ \\
Traffic type & Full-buffer \\
Number of Tx/Rx antennas & $2 \times 2$ \\
MAC protocol & $\mathrm{CSMA} / \mathrm{CA}$ \\
\hline
\end{tabular}

The LTE frame is divided into 5 uplink subframes and 5 downlink subframes. In each subframe, the duration is $1 \mathrm{~ms}$. According to the Channel Quality Indicator (CQI) of LTE UEs, we choose the Modulation and Coding Scheme (MCS). And LTE resources are allocated with a proportional fair scheduler. Error correction, i.e., Chase combining Hybrid Automatic Repeat Request (HARQ), is used for LTE packets. The frequency resolution of simulator is $180 \mathrm{kHz}$, which is in correspondence with a PRB in LTE. The operation of the Wi$\mathrm{Fi}$ network is based on Distributed Coordination Function (DCF) mode. And to in the event of packet reception error, we adopt ACK signaling and retransmission. Two energy thresholds for channel vacancy detection are taken into consideration by CSMA with collision avoidance (CSMA/CA) protocol. And they are $-63 \mathrm{dBm}$ for LTE transmissions and $80 \mathrm{dBm}$ for Wi-Fi transmissions.

In the simulation, nodes of both Wi-Fi and LTE networks are randomly distributed among the rooms, Since it is unreasonable to assume a deployment of two concurrent networks in a room exists, there is no mixed LTE and Wi-Fi deployment occurring in a single room. Wi-Fi Stations and LTE UEs are referenced as STAs, while Wi-Fi Access Points and LTE eNode base stations as APs. In both LTE and Wi-Fi, STAs are assigned to the best-serving APs if we only consider shadow fading and path loss.

\section{Simulation RESUlts}

The Wi-Fi only and LTE only configurations are taken as reference cases. In terms of the LTE/Wi-Fi coexistence, we take two sets of simulations into account so as to assess the performance of the LTE UL power control which is shown in table 1 . In the first set of simulations, there are 4 Access Points (APs) of each technology deployed among the 20 room office scenario. And in the second, 10 APs are deployed on the same scenario. In the above cases, we evaluate the performance by tracking mean user throughput for $10 \mathrm{Wi}-\mathrm{Fi}$ Stations (STAs) and 25 STAs of LTE and then Wi-Fi.

As demonstrated in Fig. 5, we can see the simulation results in the situation of LTE only and Wi-Fi only deployments, and the two technologies coexisting. The observing results have similarity with those given in [12]. In the latter ones, there's no LTE power control being considered. In other words, Wi-Fi interference exerts slight influence on LTE, but the degradation of Wi-Fi throughput can be serious according to the scenario. For the deployment of 4 APs of Wi-Fi only, mean user throughput turns out to be $24.2 \mathrm{Mbps}$ for 10 STAs and 8.4 
Mbps for 25 STAs. While coexisting with LTE, Wi-Fi throughput is 12.8 Mbps and 4.4 Mbps, respectively. And in the two situations, Wi-Fi performance degradation is around $46 \%$. In the case of 10 AP deployment scenarios, there is a stronger interference between LTE and Wi-Fi. Wi-Fi only throughput is $30.8 \mathrm{Mbps}$ for 10 STAs and 11.4 Mbps for 25 STAs. With LTE coexisting, Wi-Fi throughput is reduced to 6.2 Mbps and 2.2 Mbps, respectively. Consequently, in the case of 10 AP deployment scenarios, Wi-Fi throughput degradation is approximately $79 \%$.

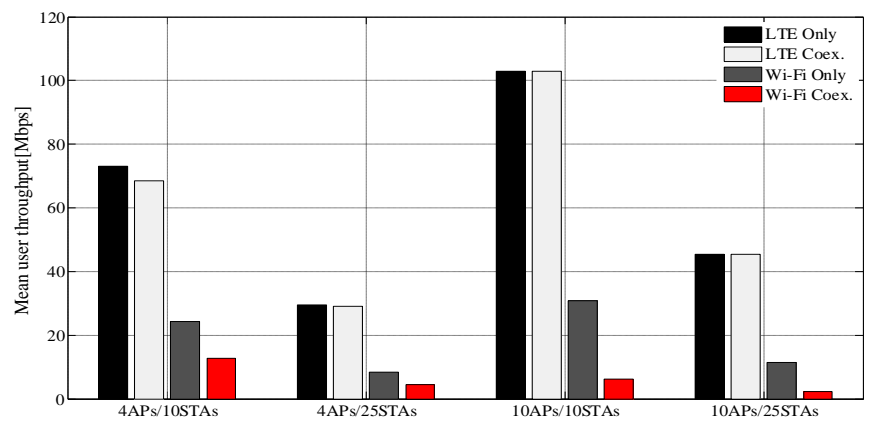

Fig. 5. Mean throughput per user for LTE only, Wi-Fi only, and both networks in coexistence.

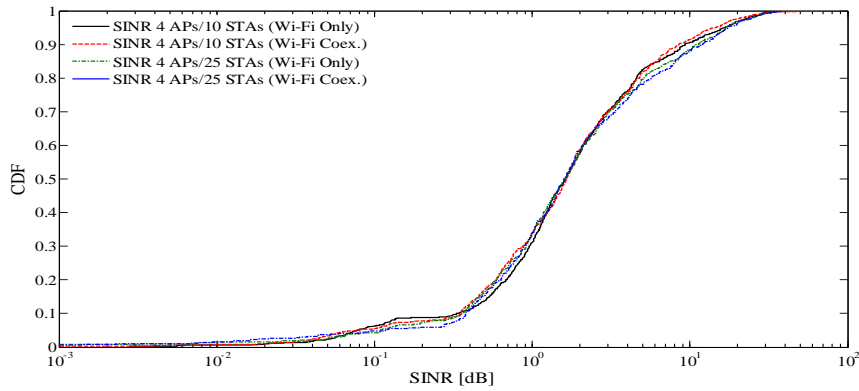

Fig. 6. Cumulative distribution function of the SINR: Sparse deployment.

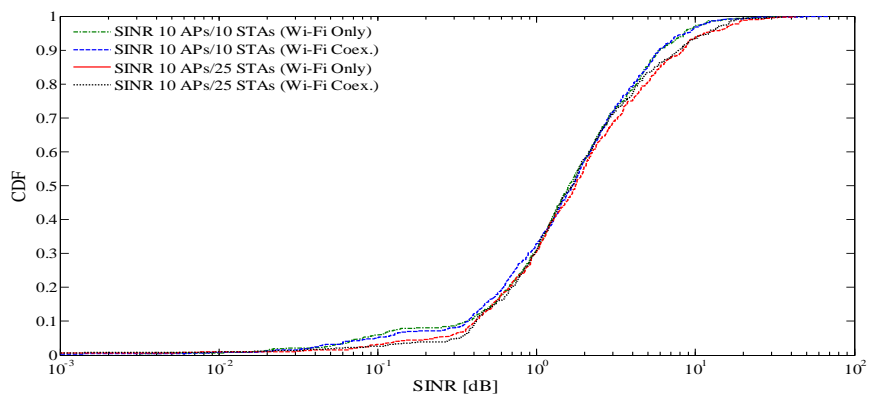

Fig. 7. Cumulative distribution function of the SINR: Dense deployment.

In order to achieve completeness, Fig. 6 shows the CDF of the UL Wi-Fi SINR for sparse deployment, and Fig. 7 demonstrates that for dense deployment. According to the results, we believe that in coexistence leads to a degradation of Wi-Fi SINR performance in the cases of both deployments. Nevertheless, we find that in some cases, such as $10 \mathrm{APs} / 10$ STAs, Wi-Fi SINR performance turns out to be alike or even better. This behavior refers to the fact that LTE transmissions can block several Wi-Fi nodes, removing them from channel contention and then minimizing collisions. This is due to that
LTE transmissions can block several Wi-Fi nodes. They are removed from channel contention and then collisions can be minimized. While LTE and Wi-Fi coexist, only few nodes are accessible to channels for a short time. In this situation, the SINR performance is not reflected on the throughput.

\section{CONCLUSIONS}

To achieve LTE/Wi-Fi coexistence in unlicensed band, we propose some schemes In this paper. According to the tests, the spectral efficiency of LTE is higher than that of $\mathrm{Wi}-\mathrm{Fi}$ in unlicensed band. Therefore, it is reasonable for LTE to compete for using unlicensed band. Simulation results show that the communication rate of users and the cell throughput are significantly improved by using licensed band and unlicensed band in LTE network. Meanwhile, the performance of Wi-Fi has slightly degraded. The range of the performance degradation of $\mathrm{Wi}-\mathrm{Fi}$ is narrower than that of the performance promotion of LTE. Hence, the performance promotion of overall system can be achieved.

\section{Acknowledgment}

We would like to thank the anonymous referees whose comments helped us to improve the presentation of this paper. This paper is funded by: Scientific and Technological Research Program of Chongqing Municipal Education Commission (KJ1501107, KJ1501105).

\section{References}

[1] Cisco Public, Cisco Visual Networking Index: "Global Mobile Data Traffic Forecast Update, 2013-2018," [EB/OL]

http://www.cisco.com/c/en/us/solutions/collateral/serviceprovider/visual-networking-index-vni/white_paper_c11-520862.html.

[2] A Ghosh, R Ratasuk, B Mondal, N. Mangalvedhe, and T. Thomas, "LTE-Advanced: next-generation wireless broadband technology," IEEE Wireless Communications, vol. 17, pp. 11-22, March 2010.

[3] H. Bogucka, P. Kryszkiewicz, and A. Kliks, "Dynamic spectrum aggregation for future $5 \mathrm{G}$ communications," IEEE Communications Magazine, vol. 53, pp. 35-43, May 2015.

[4] A. Al-Dulaimi, S. Al-Rubaye, Q. Ni, and E. Sousa, "5G communications race: pursuit of more capacity triggers LTE in unlicensed band," IEEE Vehicular Technology Magazine, vol. 10, pp. 43-51, January 2015.

[5] R. Ratasuk, M. A. Uusitalo, N. Mangalvedhe, A. Sorri, S. Iraji, C. Wijting, and A. Ghosh, "License-exempt LTE deployment in heterogeneous network," Proceedings of the 9th International Symposium Wireless Communication Systems, pp. 246-250, Paris French, August 2012.

[6] 3GPP, RP-141615, "Scope and workplan for the study on licensedassisted Access," 2014.

[7] 3GPP, "Detailed coexistence evaluation assumptions for LAA," 2014.

[8] H. J. Zhang, X. L. Chu, W. S. Guo and S. Y. Wang, "Coexistence of WiFi and heterogeneous small cell networks sharing unlicensed spectrum," IEEE Communications Magazine, vol. 53, pp. 158-164, March 2015.

[9] LTEU- Phase0 v5 QCOM-V-ALU, 2014.

[10] Qualcomm, "Extending-the-benefits-of-LTE-advanced-to-unlicensedspectrum," 2014.

[11] Qualcomm, "LTE-unlicensed-coexistence-whitepaper," 2014.

[12] 3GPP, RP-141664, "Study on licensed-assisted access using LTE," 2014

[13] A. D. Kora, S. A. Ossoubita Pouka, and J. K. Tamgno, "Enhanced indoor propagation model for WLAN system," Proceedings of the 14th International Conference on Advanced Communication Technology, pp. 1011-1015, Pyeong Chang Korea, February 2012. 